\title{
Synthetic image generator for defocusing and astigmatic PIV/PTV
}

\author{
Rossi, Massimiliano
}

Published in:

Measurement Science and Technology

Link to article, DOI:

10.1088/1361-6501/ab42bb

Publication date:

2019

Document Version

Early version, also known as pre-print

Link back to DTU Orbit

Citation (APA):

Rossi, M. (2019). Synthetic image generator for defocusing and astigmatic PIV/PTV. Measurement Science and Technology, 31(1), [017003]. https://doi.org/10.1088/1361-6501/ab42bb

\section{General rights}

Copyright and moral rights for the publications made accessible in the public portal are retained by the authors and/or other copyright owners and it is a condition of accessing publications that users recognise and abide by the legal requirements associated with these rights.

- Users may download and print one copy of any publication from the public portal for the purpose of private study or research.

- You may not further distribute the material or use it for any profit-making activity or commercial gain

- You may freely distribute the URL identifying the publication in the public portal

If you believe that this document breaches copyright please contact us providing details, and we will remove access to the work immediately and investigate your claim. 


\title{
Synthetic image generator for defocusing and astigmatic PIV/PTV
}

\author{
Massimiliano Rossi* \\ Department of Physics, Technical University of Denmark, \\ DTU Physics Building 309, DK-2800 Kongens Lyngby, Denmark
}

\begin{abstract}
This technical note introduces a synthetic image generator (SIG), referred to as MicroSIG, to be used for particle image/tracking velocimetry (PIV/PTV) analysis involving defocusing or astigmatic particle images. Target applications are experimental setups with volume illumination and small depth of field, in which the defocusing of particles plays a major role. This includes for instance $\mu \mathrm{PIV}$ experiments or 3D PTV methods using defocusing or astigmatism to determine the out-of-plane particle position. The software uses an approximated model of spherical lens and conventional ray tracing to create realistic defocused/astigmatic particle images within a reasonable computational time. MicroSIG can be used to compare or optimize different PIV/PTV algorithms using arbitrary flows and different conditions including, non-monodispersed particles, non-uniform illumination, astigmatic optics, non-spherical particles (spheroids). A version of the software, available in Matlab and Python, can be found in the supplementary material and at this link: https://gitlab.com/defocustracking.
\end{abstract}

\section{INTRODUCTION}

Synthetic image generators (SIG) are commonly used in particle image/tracking velocimetry (PIV/PTV) research studies to create artificial sets of images that simulate experimental PIV/PTV recordings [1]. The obvious advantage of synthetic images is that the positions and displacements of particles are known a priori and different experimental conditions (such as seeding density, illumination, particle sizes) can be easily realized by changing few parameters in the code. Synthetic images have been extensively used in PIV/PTV research: from classical studies to determine the precision of the method and the optimal design rules [2-5], to more recent works assessing the resolution and accuracy of TomographicPIV [6] or single-pixel correlation methods [7], just to give few examples. An attempt to provide a standardized tool for generating synthetic images was the EUROPIV SIG [8], developed within the EUROPIV 2 project. Standardized synthetic images were used in the various editions of the PIV Challenge, a world-wide periodical initiative to assess the current state of the art of PIV techniques [9].

Most SIGs use 2D Gaussian functions to model particle images, which is the classical approach in PIV analysis [10]. This approach provides realistic results in case of in-focus particles with diameter of few pixels, which represents a large part of conventional and multi-cameras PIV/PTV experiments. On the other hand, for methods using large magnification optics and volume illumination, such as $\mu \mathrm{PIV}$ [11] or single-camera 3D methods $[12,13]$, it is necessary to model also particles which are out of focus. Several models can be used for this purpose, for instance using again the 2D Gaussian function approximation [14, 15], or the Huygens-Fresnel diffraction integral [16]. All these approaches, however, neglect the spherical aberration that has a significant effect on

* rossi@fysik.dtu.dk these setups. Spherical aberration is a consequence of the spherical shape of real lenses which are in general not able to focus rays on a single spot (see Fig. 1).

This technical note introduces a SIG specifically designed to provide realistic images of in- and out-of-focus particles taken with large magnification optics. The SIG consists of a ready-to-use software, named MicroSIG, that is available in Matlab and Python. MicroSIG relies on the following assumptions:

- Particles are sphere or spheroids emitting light uniformly from their surface (like fluorescent particles).

- The objective lens is a spherical lens infinitely thin.

- Only geometric optics is used (diffraction is neglected).

MicroSIG can be used as a standardized tool to test and develop PIV and PTV methods dealing with defocused particle images. Additionally MicroSIG allows

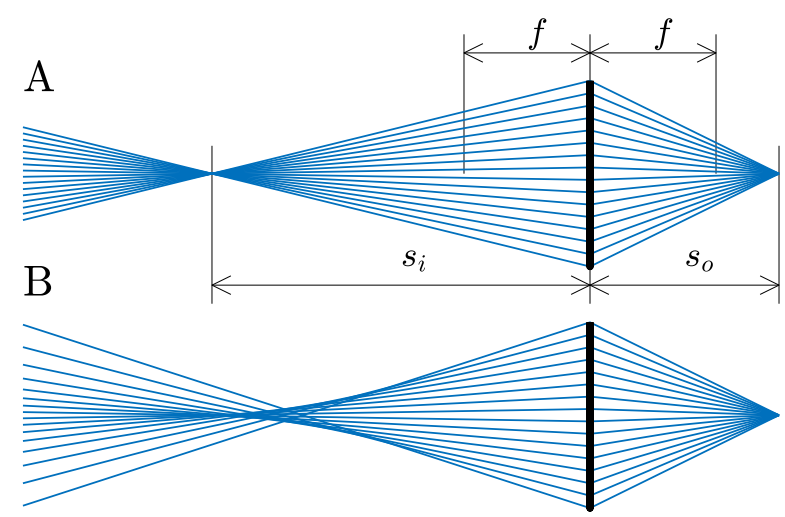

FIG. 1. (A) Ray tracing using a perfect thin lens: Rays originated from a point source at distance $s_{o}$ from the lens converge to a single point at distance $s_{i}$, following the formula $1 / s_{i}+1 / s_{o}=1 / f$, being $f$ the focal lens of the lens. (B) Ray tracing using a spherical lens: Rays originated from a point source do not converge to a single point. 

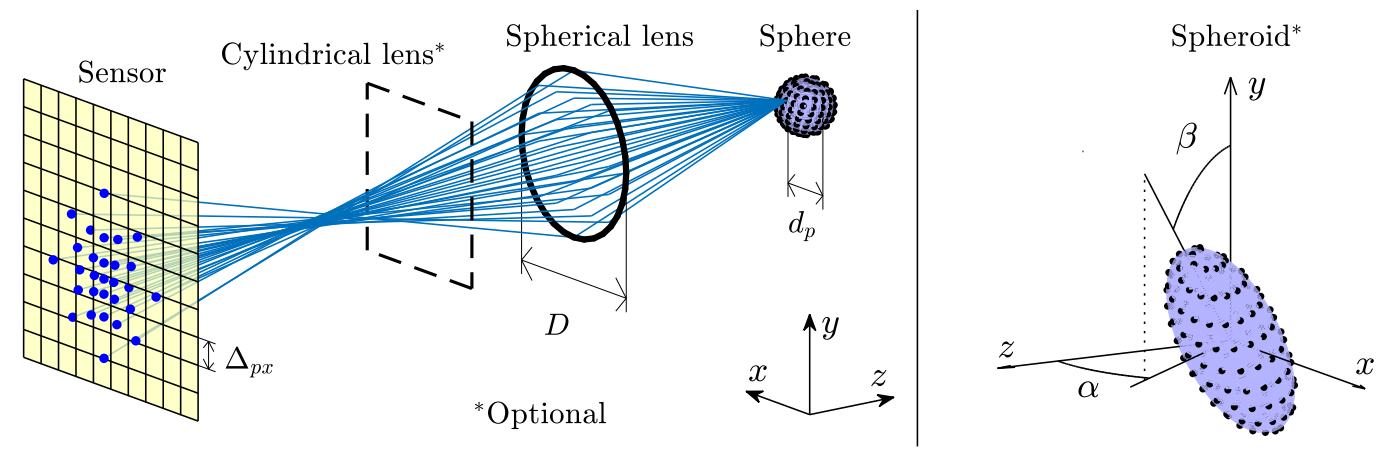

FIG. 2. General concept of MicroSIG. Tracer particles are spheres or spheroids, with light point-sources uniformly distributed on the surface. The path of each ray going through a spherical lens (and optionally also a cylindrical lens) is calculated. The intensity of one pixel is calculated as the count of rays hitting its area. A sketch of a spheroid particle with $e_{p}=3$ and its respective orientation angles $\alpha$ and $\beta$ is presented on the right panel.

to simulate spheroidal particles and astigmatic optics. Astigmatic imaging emerged as one robust method for 3D PTV $[15,17]$ and it has been successfully applied in many applications [18-21], however up to now a standardized SIG suitable for astigmatic particle images was not available yet.

\section{IMPLEMENTATION}

MicroSIG uses a three-dimensional ray-tracing approach, needed to deal with non-spherical particles and astigmatic lenses (systems that are not axisymmetric). The ray-tracing algorithm uses a matrix implementation, in which each ray crossing a plane perpendicular to the optical axis is described by a four-dimensional vector [22]:

$$
\boldsymbol{r}=\left[\begin{array}{l}
q_{x} \\
q_{y} \\
p_{x} \\
p_{y}
\end{array}\right]
$$

where the vector $\boldsymbol{q}=\left(q_{x}, q_{y}\right)$ represents the position of the intersection point of the ray with the plane, and the vector $\boldsymbol{p}=\left(p_{x}, p_{y}\right)$ its orientation.

If the ray travels undisturbed, the ray vector $\boldsymbol{r}^{\prime}$ corresponding to a different plane can be calculated as:

$$
\boldsymbol{r}^{\prime}=\left[\begin{array}{c}
q_{x}+t p_{x} \\
q_{y}+t p_{y} \\
p_{x} \\
p_{y}
\end{array}\right]=\left[\begin{array}{llll}
1 & 0 & t & 0 \\
0 & 1 & 0 & t \\
0 & 0 & 1 & 0 \\
0 & 0 & 0 & 1
\end{array}\right]\left[\begin{array}{c}
q_{x} \\
q_{y} \\
p_{x} \\
p_{y}
\end{array}\right]=\boldsymbol{T r}
$$

where $t$ is the distance between the two planes. A lens can be considered as a function $\lambda$, depending on the focal length $f$, that modifies the orientation vector:

$$
\boldsymbol{r}^{\prime}=\left[\begin{array}{c}
q_{x} \\
q_{y} \\
p_{x}^{\prime} \\
p_{y}^{\prime}
\end{array}\right]=\lambda(\boldsymbol{r}, f)
$$

In MicroSIG, the function $\lambda$ is constructed to simulate a infinite-thin, biconvex spherical lens. Rays are deflected using the Snell's law, first with respect to the air-glass interface, and then to the glass-air interface. The radius of curvature $R$ of the interface is calculated from the lensmaker's equation for thin lens:

$$
R=2\left(n_{l} / n_{m}-1\right) f
$$

where $n_{l}$ and $n_{m}$ are the refractive index of the lens and of the lens immersion medium, respectively.

The first step for creating a particle image is to generate a discrete light field (i.e. a discrete ensemble of ray vectors) originated by a sphere (or spheroid) lying at a certain distance $z$ from the lens. Practically, a number $N_{s}$ of equally-spaced points are located on the particle surface, and for each point a number $N_{r}$ of ray vectors with random orientation is created. In this way, the discrete light field $\boldsymbol{r}_{i}$ is created, with $i=1,2, . ., N$ and $N=N_{s} \cdot N_{r}$.

The light field is projected into the sensor plane with the following transformations:

$$
\begin{aligned}
\boldsymbol{r}_{i}{ }^{\prime} & =\boldsymbol{T}_{1} \boldsymbol{r}_{i}, & & i=1,2, . ., N \\
\boldsymbol{r}_{j}{ }^{\prime \prime} & =\lambda\left(\boldsymbol{r}_{j}{ }^{\prime}, f\right), & & j \subseteq i \\
\boldsymbol{r}_{k}{ }^{\mathrm{ccd}} & =\boldsymbol{T}_{2} \boldsymbol{r}_{k}{ }^{\prime \prime}, & & k \subseteq j
\end{aligned}
$$

where $\boldsymbol{T}_{1}$ is the translation matrix from the particle to the lens, and $\boldsymbol{T}_{2}$ from the lens to the sensor. The index $j$ indicates the rays that hit the lens and are a subset of $i, k$ indicates the rays that hit the sensor and are a subset of $j$. The sensor is composed by squared pixels with fill factor equal to 1 . Finally, the intensity (counts) measured by a single pixels is calculated by the number of rays hitting its area.

If the astigmatic optics is activated, Eq. (7) is substituted by:

$$
\boldsymbol{r}_{k}{ }^{\mathrm{ccd}}=\boldsymbol{T}_{2} \boldsymbol{L}_{c y l} \boldsymbol{T}_{2 c y l} \boldsymbol{r}_{k}^{\prime \prime}, \quad k \subseteq j
$$


where $\boldsymbol{L}_{c y l}$ is a linear transformation corresponding to a perfect cylindrical lens with focal length $f_{c y l}$ :

$$
\boldsymbol{L}_{c y l}=\left[\begin{array}{cccc}
1 & 0 & 0 & 0 \\
0 & 1 & 0 & 0 \\
-1 / f_{c y l} & 0 & 1 & 0 \\
0 & 0 & 0 & 1
\end{array}\right]
$$

and $\boldsymbol{T}_{2 c y l}$ and $\boldsymbol{T}_{2}$ are translation matrices from the lens to the cylindrical lens, and from the cylindrical lens to the sensor. The astigmatic aberration created by the cylindrical lens can be adjusted either by modifying its position or its focal length [15]. To reduce complexity, it was decided to arbitrarily place the cylindrical lens at $2 / 3$ the distance between the main lens and the sensor, and leave as free parameter only the focal length $f_{c y l}$.

\section{THE MICROSIG SOFTWARE}

The MicroSIG software takes as first input a setting file, which contains a list of 14 parameters defining the optics and the camera settings:

- magnification: Magnification of the optics.

- numerical_aperture: Numerical aperture of the optics.

- focal_length: Focal length of the virtual spherical lens.

- ri_medium: Refractive index of the immersion medium of the lens, typically air.

- ri_lens: Refractive index of the of the lens.

- pixel_size: Size of the pixels in $\mu \mathrm{m}$.

- pixel_dim_x: Sensor width in pixels.

- pixel_dim_y: Sensor height in pixels.

- background_mean: Constant image background value.

- background_noise: Standard deviation of the Gaussian noise added to the images.

- points_per_pixel: Number of point sources per unit area, calculated as $N_{s}$ divided by the theoretical area of an in-focus particle image (optimal values: 10-20).

- n_rays: Number of rays on each point source, corresponding to $N_{r}$ (optimal values: 100-500).

- gain: Multiplicative factor to adjust the final image intensity.

- cyl_focal_length: Focal length of the virtual cylindrical lens. If set to 0 , no astigmatism is present.

focal_length and cyl_focal_length are virtual parameters of the optical model and must be determined empirically. Recommended values, obtained from comparison with experimental images in the literature [13, 23] are 350 $\mu \mathrm{m}$ and $4000 \mu \mathrm{m}$, respectively. Examples are provided in the next section.

Subsequent inputs are the data files, one for each image to generate. The data files must be provided in ASCII format and are row arranged (one particle, one line).

MicroSIG allows four different input formats:

- $x, y, z, d_{p}$

- $x, y, z, d_{p}, c_{i n t}$

- $x, y, z, d_{p}, e_{p}, \alpha, \beta$

- $x, y, z, d_{p}, e_{p}, \alpha, \beta, c_{i n t}$
For each particle there are four mandatory variables: $x, y, z$ and $d_{p}$. $x$ and $y$ are the particle's center coordinates in the image and are given in pixels as units. $z$ is the out-of-plane component and is given in $\mu \mathrm{m}$. $z=0$ corresponds to the theoretical coordinate of the focal plane, which however may not correspond to the position where the particle looks more in focus due to the spherical aberration. $d_{p}$ is the particle size (in $\left.\mu \mathrm{m}\right)$.

To simulate spheroidal particles (i.e. ellipsoids of revolution), three additional parameters are needed: $e_{p}$, a factor which indicates the elongation along one axis of the particle (oblates for $e_{p}<1$, prolates $e_{p}>1$ ), $\alpha$ and $\beta$, which are the orientation angles as defined in Fig. 2. Finally, $c_{i n t}$ is an optional multiplication factor to simulate light intensity inhomogeneities.

\section{RESULTS}

Two examples of comparison between experimental and simulated particle image scans along the $z$-direction are reported in Fig. 3. In the first example in Fig. 3A, the experimental data are taken from [13] and correspond to a 2- $\mu$ m-diameter particle observed with a microscope objective with magnification $M=10 \times$ and numerical aperture $\mathrm{NA}=0.3$. The scan is taken across a total height of $120 \mu \mathrm{m}$, with steps of $2 \mu \mathrm{m}$ for a total of $61 \mathrm{im}-$ ages. The corresponding synthetic images were obtained using the setting parameters listed in Table I (Setting 1). The data files use the format $\left[x, y, z, d_{p}\right]$, with $d_{p}=2$ $\mu \mathrm{m}$ and $z$ ranging from -80 to $40 \mu \mathrm{m}$ with steps of $2 \mu \mathrm{m}$. It should be noted that MicroSIG considers a particle at $z=0$ always at the theoretical focal plane, and that this does not correspond exactly to the point of maximum intensity as a consequence of the spherical aberration (as shown in Fig. 3C). Also the choice of focal_length affects the rate of change of defocusing across the $z$-direction.

The second example is shown in Fig. 3B and corresponds to a 1- $\mu \mathrm{m}$-diameter particle observed with a mi-

\begin{tabular}{lllll}
\hline Parameter & Unit & \multicolumn{3}{l}{ Setting } \\
\cline { 3 - 5 } & & 1 & $1 \mathrm{a}$ & 2 \\
\hline magnification & - & 10 & 10 & 63 \\
numerical_aperture & - & 0.3 & 0.3 & 0.75 \\
focal_length & $\mu \mathrm{m}$ & 350 & 350 & 350 \\
ri_medium & - & 1 & 1 & 1 \\
ri_lens & - & 1.5 & 1.5 & 1.5 \\
\hline pixel_size & $\mu \mathrm{m}$ & 6.45 & 6.45 & 6.45 \\
pixel_dim_x & pixels & 752 & 752 & 141 \\
pixel_dim_y & pixels & 366 & 366 & 141 \\
background_mean & counts & 350 & 350 & 52 \\
background_noise & counts & 10 & 10 & 5 \\
\hline points_per_pixel & $1 /$ pixels & 18 & 18 & 12 \\
n_rays & - & 500 & 500 & 250 \\
gain & - & 3.2 & 3.2 & 1.1 \\
\hline cyl_focal_length & $\mu m$ & 0 & 4000 & 0 \\
\hline
\end{tabular}

TABLE I. Values for the setting files used in the simulations in Section IV 
A

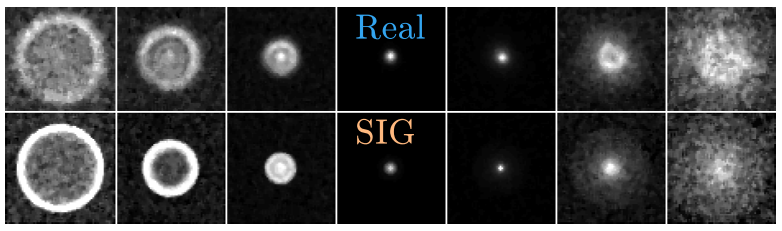

$\mathrm{B}$

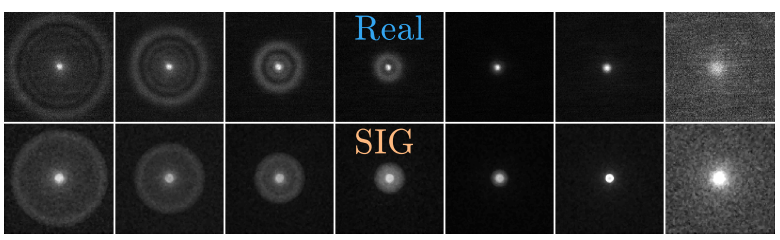

C
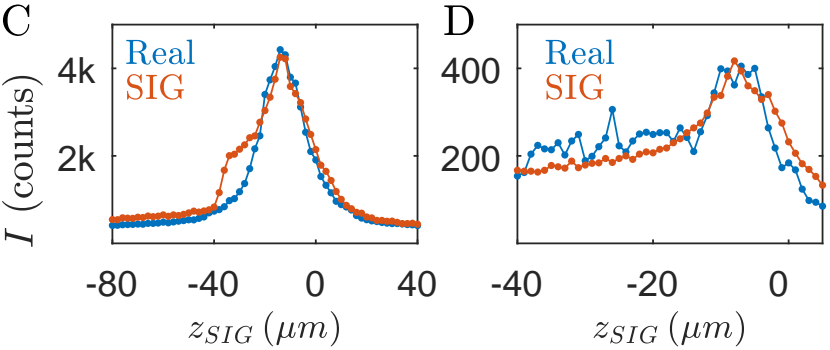

FIG. 3. Comparison between experimental and simulated particle image scans. (A) Real and synthetic images of $2-\mu \mathrm{m}$ spheres taken with a $10 \times / 0.3$ objective lens. (B) Real and synthetic images of $1-\mu \mathrm{m}$ spheres taken with a $63 \times / 0.75$ objective lens. (C) and (D) Corresponding maximum particle image intensity across the $z$ direction.

croscope objective lens with $M=63 \times$ and NA $=0.75$. The scan is taken across a total height of $45 \mu \mathrm{m}$, with steps of $1 \mu \mathrm{m}$ for a total of 46 images. The experimental data are taken from [23]. The simulated images are taken using the setting parameters listed in Table I (Setting 2) and $z$ ranging from -40 to $5 \mu \mathrm{m}$ with steps of $1 \mu \mathrm{m}$. For both examples, a good qualitative and quantitative agreement between experimental and simulated images can be observed. Some finer structures, originated from the diffraction patterns, cannot clearly be recreated by MicroSIG, however the overall structure of the defocused images is well reproduced.

Further examples are shown in Fig. 4. Fig. 4A shows experimental and simulated images of 2 - $\mu \mathrm{m}$ particles randomly distributed in a $100-\mu \mathrm{m}$-height microchannel. Fig. $4 \mathrm{~B}$ shows the same case but this time using astigmatic optics. In both cases the experimental images are taken from [13] and more detail about the experimental setup can be found there. The synthetic images were obtained using the settings 1 and 1a in Table I. For the astigmatic case, it should be noted that the value cyl_focal_length $=4000 \mu \mathrm{m}$ is a virtual parameter that cannot directly be related to the focal length of the cylindrical lens employed in the experiment $(300 \mathrm{~mm})$. Finally, Fig. 4C shows simulations of spheroidal particles with different positions and orientations. This feature allows to develop and test new algorithms able to recover the position and orientation of non-spherical objects, which are
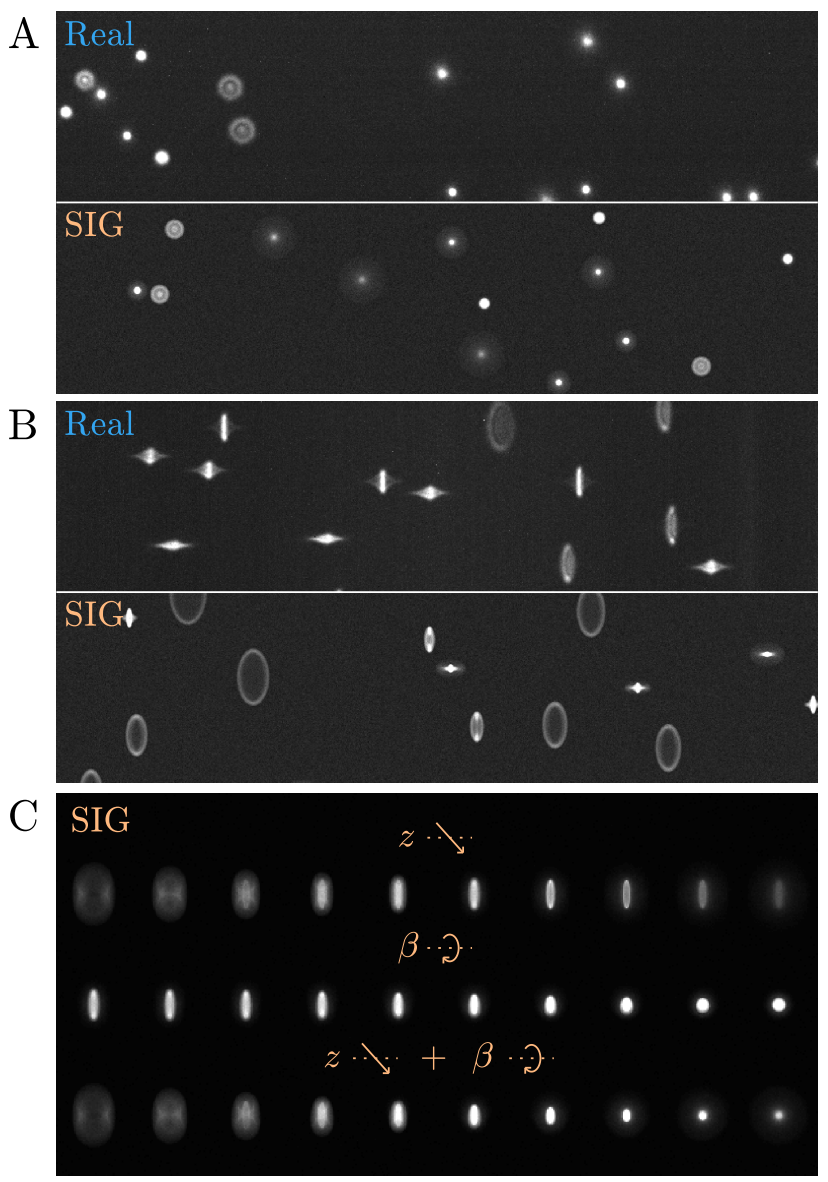

FIG. 4. (A) Real and synthetic images of 2- $\mu \mathrm{m}$ particles randomly distributed in a 100- $\mu$ m-height microchannel observed with a $10 \times / 0.3$ objective lens, and (B) same setup with in addition an astigmatic optics. (C) Simulations of spheroidal particles with different positions and orientations.

relevant in many fields from cell measurements [24, 25] to anisotropic colloidal particles [26, 27].

\section{CONCLUSIONS}

This note introduces the main features of MicroSIG, a synthetic image generator (SIG) that provides realistic defocused and astigmatic particle images. MicroSIG can be used for the assessment of the uncertainty, under different conditions, of several PIV/PTV methods in which defocusing (or astigmatism) plays a role, including $\mu \mathrm{PIV}$ or 3D-PTV single-camera methods. Furhtermore, it can be used to develop methods to measure position and orientation of non-spherical objects, which is currently an open field of research in colloids or biological flows. The MicroSIG software, available in Matlab and Python, is included in the supplementary material and can be downloaded at this link: https://gitlab.com/defocustracking. 
The research leading to these results has received funding from the European Union's Horizon 2020 research and innovation programme under the Marie Sklodowska-
Curie grant agreement no. 713683 (COFUNDfellowsDTU). The author gratefully acknowledges fruitful discussions with Rune Barnkob.
[1] K. Okamoto, S. Nishio, T. Saga, and T. Kobayashi, Measurement Science and Technology 11, 685 (2000).

[2] R. D. Keane and R. J. Adrian, Measurement science and technology 1, 1202 (1990).

[3] R. D. Keane and R. J. Adrian, Applied scientific research 49, 191 (1992).

[4] J. Westerweel, Experiments in Fluids 29, S003 (2000)

[5] F. Scarano, Measurement science and technology 13, R1 (2001).

[6] K. Lynch and F. Scarano, Experiments in Fluids 56, 66 (2015).

[7] S. Scharnowski, R. Hain, and C. J. Kähler, Experiments in fluids $\mathbf{5 2}, 985$ (2012).

[8] B. Lecordier and J. Westerweel, in Particle image velocimetry: recent improvements (Springer, 2004) pp. 145161.

[9] C. J. Kähler, T. Astarita, P. P. Vlachos, J. Sakakibara, R. Hain, S. Discetti, R. La Foy, and C. Cierpka, Experiments in Fluids 57, 97 (2016)

[10] R. J. Adrian and C.-S. Yao, Applied optics 24, 44 (1985).

[11] J. G. Santiago, S. T. Wereley, C. D. Meinhart, D. Beebe, and R. J. Adrian, Experiments in fluids 25, 316 (1998).

[12] C. Cierpka and C. Kähler, Journal of visualization 15, 1 (2012).

[13] R. Barnkob, C. J. Kähler, and M. Rossi, Lab on a Chip 15, 3556 (2015)

[14] M. Olsen and R. Adrian, Experiments in fluids 29, S166 (2000).

[15] M. Rossi and C. J. Kähler, Experiments in fluids 55, 1809
(2014).

[16] T. Fuchs, R. Hain, and C. J. Kähler, Optics letters 39 , 1298 (2014).

[17] C. Cierpka, R. Segura, R. Hain, and C. J. Kähler, Measurement Science and Technology 21, 045401 (2010).

[18] W. Qiu, J. T. Karlsen, H. Bruus, and P. Augustsson, Physical Review Applied 11, 024018 (2019).

[19] M. Rossi, A. Marin, N. Cevheri, C. J. Kähler, and M. Yoda, Microfluidics and nanofluidics 23, 67 (2019).

[20] F. Kiebert, S. Wege, J. Massing, J. König, C. Cierpka, R. Weser, and H. Schmidt, Lab on a Chip 17, 2104 (2017).

[21] R. Barnkob, N. Nama, L. Ren, T. J. Huang, F. Costanzo, and C. J. Kähler, Physical Review Applied 9, 014027 (2018).

[22] A. Gerrard and J. M. Burch, Introduction to matrix methods in optics (Courier Corporation, 1994).

[23] M. Rossi, R. Segura, C. Cierpka, and C. J. Kähler, Experiments in fluids 52, 1063 (2012).

[24] K. Taute, S. Gude, S. Tans, and T. Shimizu, Nature communications 6, 8776 (2015)

[25] J. S. Guasto, R. Rusconi, and R. Stocker, Annual Review of Fluid Mechanics 44, 373 (2012).

[26] J.-C. Loudet, A. M. Alsayed, J. Zhang, and A. G. Yodh, Physical review letters 94, 018301 (2005).

[27] M. Cavallaro, L. Botto, E. P. Lewandowski, M. Wang, and K. J. Stebe, Proceedings of the National Academy of Sciences 108, 20923 (2011). 